\title{
System Identification and Automatic Mass Balancing of Ground-Based Three-Axis Spacecraft Simulator
}

\author{
Jae-Jun Kim* and Brij N. Agrawal ${ }^{\dagger}$ \\ Department of Mechanical and Astronautical Engineering \\ Naval Postgraduate School, Monterey, CA 93943
}

\begin{abstract}
The ground-based spacecraft simulator is a useful tool to develop and verify new control laws required by modern spacecraft applications. In order to simulate the space environment with the ground-based spacecraft simulator, the effects of gravity should be minimized. In this paper, a three-axis rotational rigid spacecraft simulator on a spherical air-bearing system is considered. The method of estimating the inertial properties of a spacecraft as well as the location of the center of mass is presented with the batch leastsquare estimation. The identified center of mass location is used to actuate the automatic mass balancing system to compensate for the center of mass offset. Adaptive control of the automatic mass balancing system is also presented when the balancing masses are actuated in real-time to eliminate the center of mass offset from the center of rotation. The proposed technique is implemented on the Three Axis Simulator 2 (TAS2) which is a ground-based experimental testbed for the Bifocal Relay Mirror Spacecraft (BRMS).
\end{abstract}

\section{Introduction}

Ground simulation and testing of spacecraft attitude dynamics is highly desirable because it is extremely difficult to test and reconfigure the system once the vehicle is in the space. Rigorous ground testing of the spacecraft attitude control system will significantly reduce the risk to the project. Ground-based spacecraft testbeds often utilize air-bearings to simulate frictionless and micro-gravity space environments. Various airbearing-based spacecraft simulators have been developed in the past, and the historical review is presented in. ${ }^{1}$ As in reference, ${ }^{1}$ there are two types of air-bearing systems. One is the planar air-bearing system which is capable of providing one rotational and two translational degrees of freedom. The planar motion airbearing testbeds are often used for simulations of formation flying, rendezvous and docking. The other type is the spherical air-bearing system for three-dimensional rotational motion. Spherical air-bearing systems are widely used for spacecraft attitude dynamics research. However, there are various disturbance elements with the spherical air-bearing testbeds, which limit the simulation of the space environment during spacecraft attitude dynamics experiment. ${ }^{1}$ Among the disturbances, gravity torque effects are the most significant and the most observed behavior.

A spherical air-bearing-based spacecraft testbed requires the center of mass location of the spacecraft be accurately aligned with the center of rotation of the spacecraft. Manual balancing of the testbed is a time consuming process with limited accuracy. When the center of mass is located below the center of rotation, the testbed behaves like a pendulum. Since the pendulum system has a stable equilibrium point when the center of mass is located along the gravity vector, the balance of the system cannot be guaranteed just by observing the testbed maintaining one fixed attitude. In order to overcome the difficulty of manual balancing, an automatic mass balancing system has been employed in the recent testbed. ${ }^{3}$ In reference, ${ }^{3}$ the automatic mass balancing system is composed of three masses on linear stages, which can alter their positions relative to the spacecraft body. The set of equilibrium points are recorded for different locations of these balancing masses. Since these equilibrium points indicate the center of mass is located along the

* NRC Research Associate, Department of Mechanical and Astronautical Engineering, Naval Postgraduate School, Monterey, CA 93943, Member AIAA.

${ }^{\dagger}$ Distinguished Professor, Department of Mechanical and Astronautical Engineering, Naval Postgraduate School, Monterey, CA 93943, Associate Fellow AIAA. 
gravity vector, the center of mass can be estimated using these equilibrium points. In order to achieve better accuracy in estimating the location of the center of mass, it is desirable to record a lot of equilibrium points. Therefore, this method may require a lot of time.

In this paper, batch linear least-square estimation of the spacecraft inertia and the center of mass is considered first. The estimated center of mass is directly used for the compensation of the center of mass offset from the center of rotation. An adaptive control method for automatic mass balancing system is also developed for on-line compensation of the center of mass offset. The proposed techniques are implemented on the Three Axis Simulator 2 (TAS2) which is a spherical air-bearing-based testbed for the Bifocal Relay Mirror Spacecraft (BRMS) developed at the Naval Postgraduate School.

\section{Batch Estimation of Moment of Inertia and Center of Mass}

In this development, a rigid body three-axis spacecraft simulator with momentum exchange devices is considered. A simple equation describing the dynamics of the spacecraft simulator in the spacecraft bodyfixed coordinates is written as

$$
J \dot{\omega}+\omega \times J \omega=-\dot{\mathbf{h}}-\omega \times \mathbf{h}+\mathbf{r} \times m \mathbf{g}
$$

where, $J$ is the total moment of inertia including the momentum exchange devices, $\omega$ is the angular rate of the spacecraft, $\mathbf{h}$ is the total momentum of the momentum exchange devices, $m$ is the total mass, $\mathbf{r}$ is a constant vector from the center of rotation to the center of mass (center of mass vector in spacecraft body frame), and $\mathbf{g}$ is the gravity acceleration vector. The unknowns to be estimated in Equation 1 are the inertia matrix $J$ and the center of mass vector $\mathbf{r}$. Defining matrix $\Omega$ and vector of inertia matrix elements $\tilde{\mathbf{J}}$ as

$$
\Omega=\left[\begin{array}{cccccc}
\omega_{1} & 0 & 0 & \omega_{2} & \omega_{3} & 0 \\
0 & \omega_{2} & 0 & \omega_{1} & 0 & \omega_{3} \\
0 & 0 & \omega_{3} & 0 & \omega_{1} & \omega_{2}
\end{array}\right], \quad \tilde{\mathbf{J}}=\left[\begin{array}{llllll}
J_{x x} & J_{y y} & J_{z z} & J_{x y} & J_{x z} & J_{y z}
\end{array}\right]^{T},
$$

Equation 1 can be rewritten as

$$
\dot{\Omega} \tilde{\mathbf{J}}+\omega \times \Omega \tilde{\mathbf{J}}=-\dot{\mathbf{h}}-\omega \times \mathbf{h}-[\mathbf{g} \times] m \mathbf{r}
$$

where the cross product matrix is defined by the following relationship.

$$
[\mathbf{a} \times]=\left[\begin{array}{ccc}
0 & -a_{3} & a_{2} \\
a_{3} & 0 & -a_{1} \\
-a_{2} & a_{1} & 0
\end{array}\right], \quad \mathbf{a}=\left[\begin{array}{c}
a_{1} \\
a_{2} \\
a_{3}
\end{array}\right]
$$

Equation 3 may be written in matrix form, which is also done in ref. ${ }^{3}$

$$
\left[\begin{array}{ll}
\dot{\Omega}+\omega \times \Omega & {[\mathbf{g} \times]}
\end{array}\right]\left[\begin{array}{c}
\tilde{\mathbf{J}} \\
m \mathbf{r}
\end{array}\right]=-\dot{\mathbf{h}}-\omega \times \mathbf{h}
$$

Equation 5 requires the knowledge of $\dot{\omega}$ which requires numerical differentiation of the gyro signals. Therefore direct application of least-square method is not desirable. One method to eliminate the numerical derivative of the rate is to utilize the energy balance presented in ${ }^{5}$ and. ${ }^{6}$ Since the energy of the system is conserved, kinetic energy of the spacecraft is equal to the energy caused by the control input and gravity. In this paper, low-pass filtering is proposed. Both sides of Equation 1 are filtered through a low pass filter such that

$$
J \omega-\frac{a}{s+a}(J \omega)+\frac{1}{s+a}(\omega \times J \omega)+\frac{1}{s+a}(\mathbf{g} \times m \mathbf{r})=-\mathbf{h}+\frac{a}{s+a}(\mathbf{h})-\frac{1}{s+a}(\omega \times \mathbf{h})
$$

where $a$ determines the crossover frequency. Time and frequency domain is mix-used in Equation 6 for the convenience of the analytical derivation. From the definition of $\Omega$ and $\tilde{\mathbf{J}}$, Equation 6 can be rewritten as

$$
\left[\Omega-\frac{a}{s+a}(\Omega)+\frac{1}{s+a}([\omega \times] \Omega) \frac{1}{s+a}([\mathbf{g} \times])\right]\left[\begin{array}{c}
\tilde{\mathbf{J}} \\
m \mathbf{r}
\end{array}\right]=-\mathbf{h}+\frac{a}{s+a}(h)-\frac{1}{s+a}(\omega \times \mathbf{h})
$$


Now the equation is written as a standard form of the least-square problem such that $\Phi \mathbf{x}=\mathbf{y}$. There is a special case when $a=0$, which means that the filter is a simple integrator. In this case, the least square problem reduces to

$$
\left[\Omega+\int_{t_{0}}^{t}([\omega \times] \Omega) d t \quad \int_{t_{0}}^{t}[\mathbf{g} \times] d t\right]\left[\begin{array}{c}
\tilde{\mathbf{J}} \\
m \mathbf{r}
\end{array}\right]=-\mathbf{h}-\int_{t_{0}}^{t}(\omega \times \mathbf{h}) d t
$$

\section{Automatic Mass Balancing System and Direct Compensation of Center of Mass}

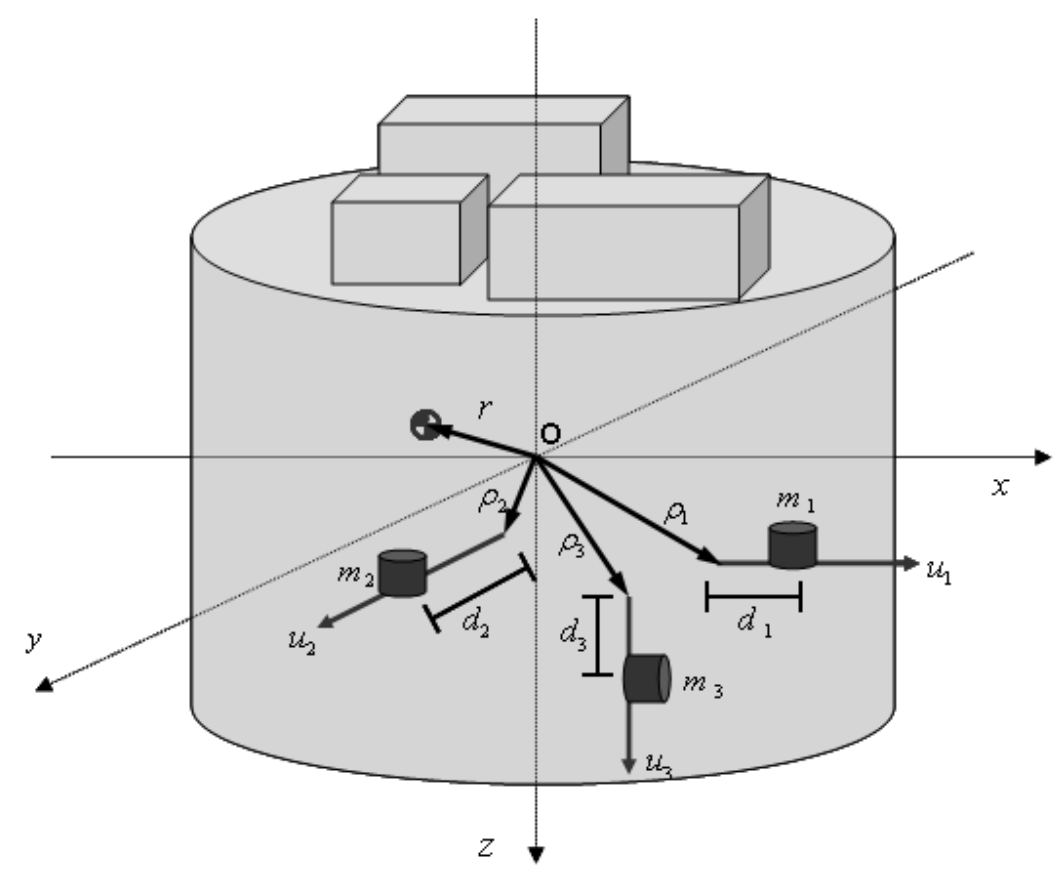

Figure 1. Automatic Mass Balancing System (AMBS)

An automatic Mass Balancing System (AMBS) is typically composed of three moving balance masses on linear stages as shown in Figure 1. The three balancing masses move along the unit vector directions represented as $\mathbf{u}_{1}, \mathbf{u}_{2}$, and $\mathbf{u}_{3}$. In Figure 1, these unit vectors are located parallel to the three axis of the spacecraft body. However, this is not a requirement for the mass balancing system. Vectors $\rho_{1}, \rho_{2}$, and $\rho_{3}$ represent the zero-locations of the balancing masses. The balancing mass displacements $d_{1}, d_{2}$, and $d_{3}$ are referenced from these zero-locations. Then, the location vector of each balancing mass can be represented as $\mathbf{R}_{i}=\rho_{i}+d_{i} \mathbf{u}_{i}$ for $i=1 \cdots 3$. The center of mass vector $\mathbf{r}$ is computed as

$$
\mathbf{r}=\frac{1}{m} \int_{B} R d m=\frac{1}{m}\left[\left(m-m_{B}\right) \mathbf{R}_{0}+\sum_{i=1}^{3} m_{i} \mathbf{R}_{i}\right]
$$

where, $m$ is the total mass of a spacecraft including balancing masses, and $m_{B}=m_{1}+m_{2}+m_{3}$ represents the sum of balancing masses, and $\mathbf{R}_{0}$ is the center of mass vector when the balancing masses are removed. When the balancing masses are moved by $\Delta d_{i}(i=1 \cdots 3)$, the new location of the center of mass becomes

$$
\mathbf{r}^{\prime}=\frac{1}{m}\left[\left(m-m_{B}\right) \mathbf{R}_{0}+\sum_{i=1}^{3} m_{i}\left(\rho_{i}+\left(d_{i}+\Delta d_{i}\right) \mathbf{u}_{i}\right)\right]
$$


The change in the center of mass vector becomes

$$
\Delta \mathbf{r}=\mathbf{r}^{\prime}-\mathbf{r}=\frac{1}{m} \sum_{i=1}^{3} m_{i} \Delta d_{i} \mathbf{u}_{i}
$$

In order to compensate the center of mass offset, $\Delta \mathbf{r}$ should be equal to $-\hat{\mathbf{r}}$, where $\hat{\mathbf{r}}$ is the estimated center of mass vector from the batch least-square estimation. Substituting $\Delta \mathbf{r}=-\hat{\mathbf{r}}$ and solving for $\Delta d_{i}$ yields

$$
\left[\begin{array}{l}
\Delta d_{1} \\
\Delta d_{2} \\
\Delta d_{3}
\end{array}\right]=-m\left[\begin{array}{lll}
m_{1} \mathbf{u}_{1} & m_{2} \mathbf{u}_{2} & m_{3} \mathbf{u}_{3}
\end{array}\right]^{-1} \hat{\mathbf{r}}
$$

The inertia of the spacecraft is also changed as a result of the center of mass offset correction. The estimated inertia matrix can be broken into two parts as

$$
\hat{J}=\hat{J}_{s}+\sum_{i=1}^{3}\left(-m_{i}\left[\mathbf{R}_{i} \times\right]\left[\mathbf{R}_{i} \times\right]\right)
$$

where, $\hat{J}_{s}$ stands for the estimated inertia matrix without balancing masses. The new inertia matrix after center of mass offset compensation becomes

$$
\hat{J}^{\prime}=\hat{J}-\sum_{i=1}^{3}\left(-m_{i}\left[\mathbf{R}_{i} \times\right]\left[\mathbf{R}_{i} \times\right]\right)+\sum_{i=1}^{3}\left(-m_{i}\left[\mathbf{R}_{i}^{\prime} \times\right]\left[\mathbf{R}_{i}^{\prime} \times\right]\right)
$$

where

$$
\mathbf{R}_{i}^{\prime}=\left(\rho_{i}+\left(d_{i}+\Delta d_{i}\right) \mathbf{u}_{i}\right)
$$

\section{Adaptive Control of Automatic Mass Balancing System}

In this section, an adaptive control method is proposed for the automatic mass balancing system. This method requires the initial estimation of the spacecraft inertia matrix with the methods presented in the previous section. However, the center of mass vector estimation is not required. The equation of the motion of the spacecraft simulator is

$$
\dot{\mathbf{H}}+[\omega \times] \mathbf{H}=m(\mathbf{r} \times \mathbf{g})
$$

The time-varying center of mass vector $\mathbf{r}(t)$ can be written as

$$
\mathbf{r}(t)=\mathbf{r}_{0}+\delta \mathbf{r}(t)
$$

where $\mathbf{r}_{0}$ represents the center of mass location vector at time zero and $\delta \mathbf{r}$ is the change of the center of mass due to the automatic mass balancing actuation. The problem is to drive the automatic mass balancing system such that the unknown $\mathbf{r}_{0}$ is compensated. When the simulator is perfectly balanced, $\delta \mathbf{r}$ becomes $-\mathbf{r}_{0}$ such that $\mathbf{r}=\mathbf{r}_{0}+\delta \mathbf{r}=0$. The total momentum in Equation 16 is written as

$$
\mathbf{H}=J \omega+\sum_{i=1}^{3} \mathbf{R}_{i} \times m_{i} \dot{\mathbf{R}}_{i}+\mathbf{h}
$$

where $\mathbf{h}$ is the momentum of the momentum exchange device. Note that the spacecraft inertia matrix $(J)$ is time-varying and is determined by the balance-mass positions. The inertia of the spacecraft without balancing masses can be written as

$$
J_{s}=J(0)-\sum_{i=1}^{3}\left(-m_{i}\left[\mathbf{R}_{i}(0) \times\right]\left[\mathbf{R}_{i}(0) \times\right]\right)
$$

Then the inertia matrix at time $t$ can be computed as

$$
J(t)=J_{s}+\sum_{i=1}^{3}\left(-m_{i}\left[\mathbf{R}_{i}(t) \times\right]\left[\mathbf{R}_{i}(t) \times\right]\right)
$$


The spacecraft equation of motion in Equation 16 can be further written as

$$
\dot{\mathbf{H}}_{s}+[\omega \times] \mathbf{H}_{s}=\tau-m[\mathbf{g} \times]\left(\mathbf{r}_{0}+\delta \mathbf{r}\right)
$$

where,

$$
\mathbf{H}_{s}=J \omega+\sum_{i=1}^{3} \mathbf{R}_{i} \times m_{i} \dot{\mathbf{R}}_{i}, \quad \tau=-\dot{\mathbf{h}}-[\omega \times] h
$$

Proposing the feedback control law as $\tau=(-K+[\omega \times]) \mathbf{H}_{s}$ with symmetric positive definite matrix $K$, the closed-loop equation of motion becomes

$$
\dot{\mathbf{H}}_{s}+K \mathbf{H}_{s}=-m[\mathbf{g} \times]\left(\mathbf{r}_{\mathbf{0}}-\delta \mathbf{r}\right)
$$

where $\delta \mathbf{r}$ is to be determined by the adaptation rule. Define the candidate Lyapunov function as

$$
V\left(\mathbf{H}_{s}, \delta \mathbf{r}\right)=\frac{1}{2} \mathbf{H}_{s}^{T} \mathbf{H}_{s}+\frac{1}{2}\left(\mathbf{r}_{\mathbf{0}}+\delta \mathbf{r}\right)^{\mathbf{T}}\left(\mathbf{r}_{\mathbf{0}}+\delta \mathbf{r}\right)
$$

The time derivative of the candidate Lyapunov function becomes

$$
\dot{V}=\mathbf{H}_{s}^{T} \dot{\mathbf{H}}_{s}+\left(r_{0}+\delta \mathbf{r}\right)^{T} \dot{\delta} \mathbf{r}=\mathbf{H}_{s}^{T}\left(-K \mathbf{H}_{s}-m[\mathbf{g} \times](\mathbf{r}+\delta \mathbf{r})\right)+\left(\mathbf{r}_{\mathbf{0}}+\delta \mathbf{r}\right)^{T} \dot{\delta} \mathbf{r}
$$

The adaptation rule is chosen as

$$
\dot{\delta} \mathbf{r}=m[\mathbf{g} \times]^{T} \mathbf{H}_{s}
$$

The adaptation rule can be further written as a function of the positions of the mass balancers for implementation purpose. It can be shown that

$$
\delta \mathbf{r}=\frac{1}{m} \sum_{i=1}^{3} m_{i} \delta d_{i} \mathbf{u}_{i}=\frac{1}{m} G \delta \mathbf{d}
$$

where $\delta \mathbf{d}=\left[\begin{array}{lll}\delta d_{1} & \delta d_{2} & \delta d_{3}\end{array}\right]^{T}$ and $G=\left[\begin{array}{lll}m_{1} \mathbf{u}_{\mathbf{1}} & m_{2} \mathbf{u}_{\mathbf{2}} & m_{3} \mathbf{u}_{\mathbf{3}}\end{array}\right]$. The time derivative of the equation yields

$$
\dot{\delta \mathbf{r}}=\frac{1}{m} G \dot{\delta} \mathbf{d}
$$

Then the adaptation rule becomes

$$
\dot{\delta \mathbf{d}}=m^{2} G^{-1}[\mathbf{g} \times]^{T} \mathbf{H}_{s}
$$

The time derivative of the candidate Lyapunov function with the proposed adaptation rule becomes

$$
\dot{V}=-\mathbf{H}_{s}^{T} K \mathbf{H}_{s}
$$

which is a negative semi-definite. Since $\delta \mathbf{r}$ and $\tau$ are bounded, $\dot{\mathbf{H}}_{s}$ is also bounded. By inspecting

$$
\ddot{V}=-2 \mathbf{H}_{s}^{T} K \dot{\mathbf{H}}_{s}
$$

$\ddot{V}$ is also bounded. Therefore, the momentum $\mathbf{H}_{s}$ is concluded to be stable and goes to zero as time goes to infinity. Since the momentum $\mathbf{H}_{s}$ goes to zero, the control input $\tau$ and mass balancing actuation $\delta \dot{\mathbf{r}}$ also becomes zero. This indicates that the total angular momentum $\left(\mathbf{H}=J \omega+\sum_{i=1}^{3} \mathbf{R}_{i} \times m_{i} \dot{\mathbf{R}}_{i}+\mathbf{h}\right)$ becomes constant. Although the total angular momentum is conserved with the proposed control method, it does not guarantee the correct estimation of $r_{0}$. Since the rank of skew symmetric matrix $[\mathbf{g} \times]$ is always two, there exists a null vector solution which makes $[\mathbf{g} \times] m\left(\mathbf{r}_{\mathbf{0}}+\delta \mathbf{r}\right)$ to be zero. From the following equation, any real $k$ will not affect the motion of the spacecraft simulator since $[\mathbf{g} \times] k \mathbf{g}=0$.

$$
\dot{\mathbf{H}}_{s}+[\omega \times] \mathbf{H}_{s}=\tau-m[\mathbf{g} \times]\left(\mathbf{r}_{0}+\delta \mathbf{r}+k \mathbf{g}\right)
$$

This corresponds to the situation where the center of mass is located along the gravity vector as shown in Figure 2. The system may be in the equilibrium state with the incorrect compensation of the center of mass offset as mentioned in the introduction section. Therefore, the system requires persistent excitation. The 


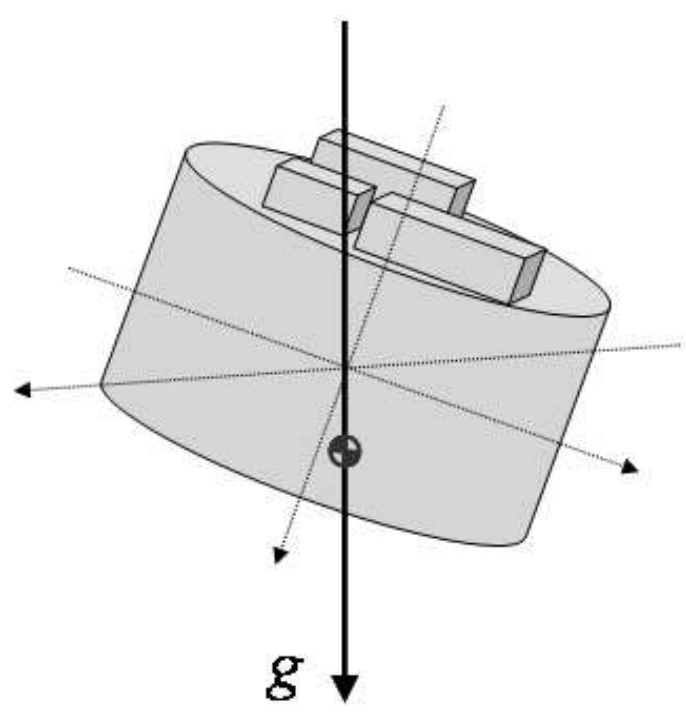

Figure 2. Null Vector Solution Example

simplest solution is to generate a desired spacecraft momentum trajectory which can provide persistent maneuvering of the spacecraft simulator. Define the desired spacecraft momentum trajectory as $\mathbf{H}_{d}$. Proposing the candidate Lyapunov function as

$$
V\left(\mathbf{H}_{s}, \delta \mathbf{r}\right)=\frac{1}{2}\left(\mathbf{H}_{s}-\mathbf{H}_{d}\right)^{T}\left(\mathbf{H}_{s}-\mathbf{H}_{d}\right)+\frac{1}{2}\left(\mathbf{r}_{0}+\delta \mathbf{r}\right)^{T}\left(\mathbf{r}_{0}+\delta \mathbf{r}\right)
$$

Time-derivative of the candidate Lyapunov function becomes

$$
\dot{V}=\left(\mathbf{H}_{s}-\mathbf{H}_{d}\right)^{T}\left(\dot{\mathbf{H}}_{s}-\dot{\mathbf{H}}_{d}\right)+\left(\mathbf{r}_{0}+\delta \mathbf{r}\right) \dot{\delta} \mathbf{r}
$$

Proposing the feedback control law as

$$
\tau=-K\left(\mathbf{H}_{s}-\mathbf{H}_{d}\right)+[\omega \times] \mathbf{H}_{s}+\dot{\mathbf{H}}_{d}
$$

Then the time-derivative of the candidate Lyapunov function becomes

$$
\dot{V}=\left(\mathbf{H}_{s}-\mathbf{H}_{d}\right)^{T}\left(-K\left(\mathbf{H}_{s}-\mathbf{H}_{d}\right)-[\mathbf{g} \times] m\left(\mathbf{r}_{0}-\delta \mathbf{r}\right)+\left(\mathbf{r}_{0}+\delta \mathbf{r}\right) \dot{\delta \mathbf{r}}\right.
$$

Let the adaptation law to be

$$
\dot{\delta \mathbf{r}}=m[\mathbf{g} \times]^{T}\left(\mathbf{H}_{s}-\mathbf{H}_{d}\right), \quad \text { equivalently } \quad \dot{\delta \mathbf{d}}=m^{2} G^{-1}[\mathbf{g} \times]^{T}\left(\mathbf{H}_{s}-\mathbf{H}_{d}\right)
$$

Then, the equation becomes

$$
\dot{V}=-\left(\mathbf{H}_{s}-\mathbf{H}_{d}\right)^{T} K\left(\mathbf{H}_{s}-\mathbf{H}_{d}\right)
$$

It can be also shown that the tracking error becomes zero as time goes to infinity. In order to verify the proposed control law, computer simulation has been performed.

The top two plots in Figure 3 show the adaptive mass balancing control simulation results without persistent excitation of the testbed. The angular momentum of the spacecraft (shown in top left plot) becomes zero as the center of mass offset is compensated using the adaptation law. However, the converged center of mass offset (shown in top right plot) did not show the correct results. The resulting center of mass becomes the null space solution of the matrix $[\mathbf{g} \times]$. With persistent excitation (shown in bottom plots), the tracking error becomes zero and the center of mass offset becomes also zero. Therefore, the simulation result verified the importance of the persistent excitation during the adaptive control of the automatic mass balancing system. 

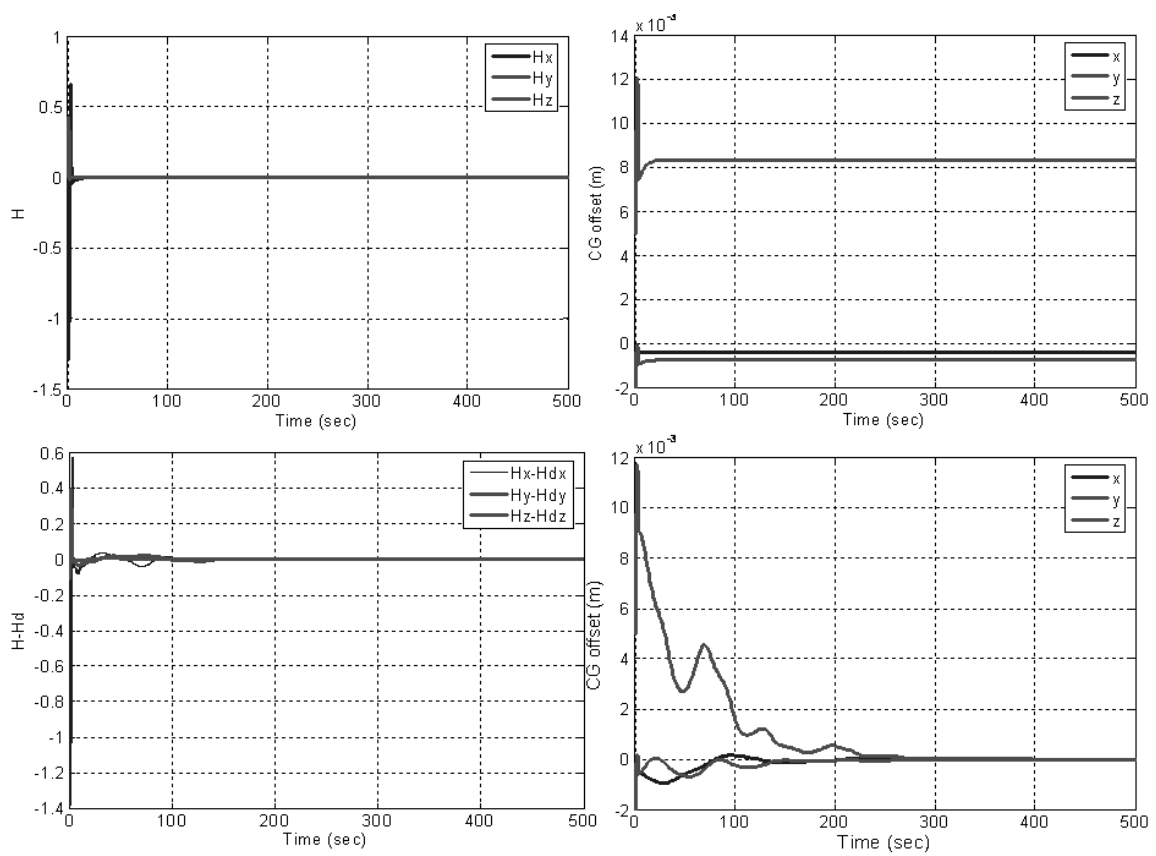

Figure 3. Adaptive Mass Balancing Control Simulation Results

\section{Experiment with the Bifocal Relay Mirror Spacecraft Simulator}

Bifocal Relay Mirror Spacecraft (BRMS), whose concept is shown in Figure 4, is composed of two optically coupled telescopes used to redirect laser light from ground-based, aircraft-based or spacecraft based lasers to distant points on the earth or in Space. The receiver telescope captures the incoming laser beam and the transmit telescope directs the beam to the desired target point. The BRMS consists of single axis gimballed receive and transmit telescopes with 1.64 meter diameter primary mirrors and fast steering mirrors for fine beam control. The transmit telescope is attached to a majority of the spacecraft bus subsystems including the attitude control sensors and actuators. Figure 5 is a Three-Axis Simulator 2 (TAS2) developed at the Naval

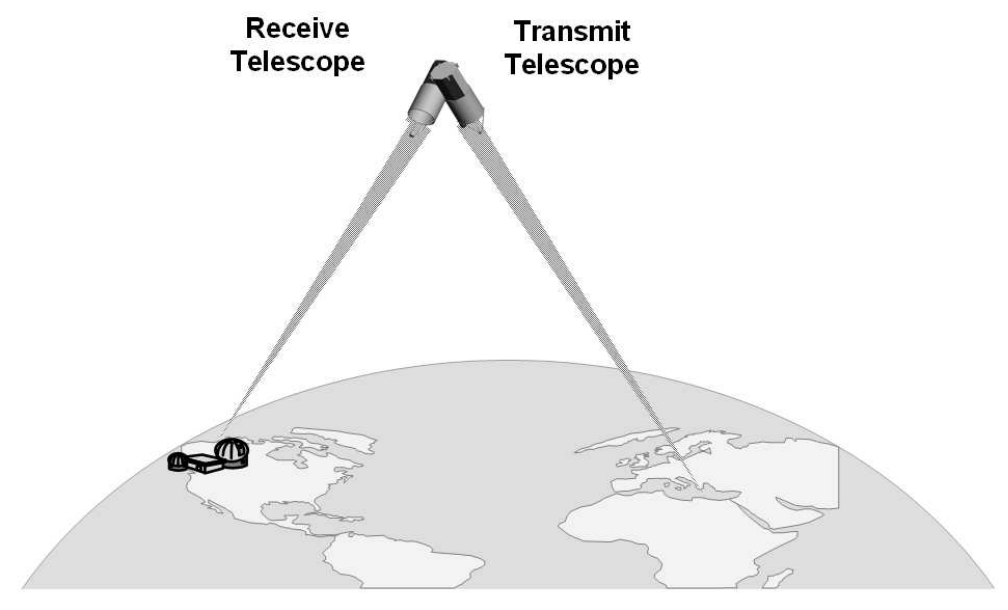

Figure 4. Bifocal Relay Mirror Spacecraft Concept

Postgraduate School for simulations of the BRMS. Currently, three Control Moment Gyroscopes (CMGs) are served as a primary actuator of the spacecraft simulator. The change in the direction of the momentum built by the CMG flywheel has the amplification effect of the torque generated. Therefore, high torque can 


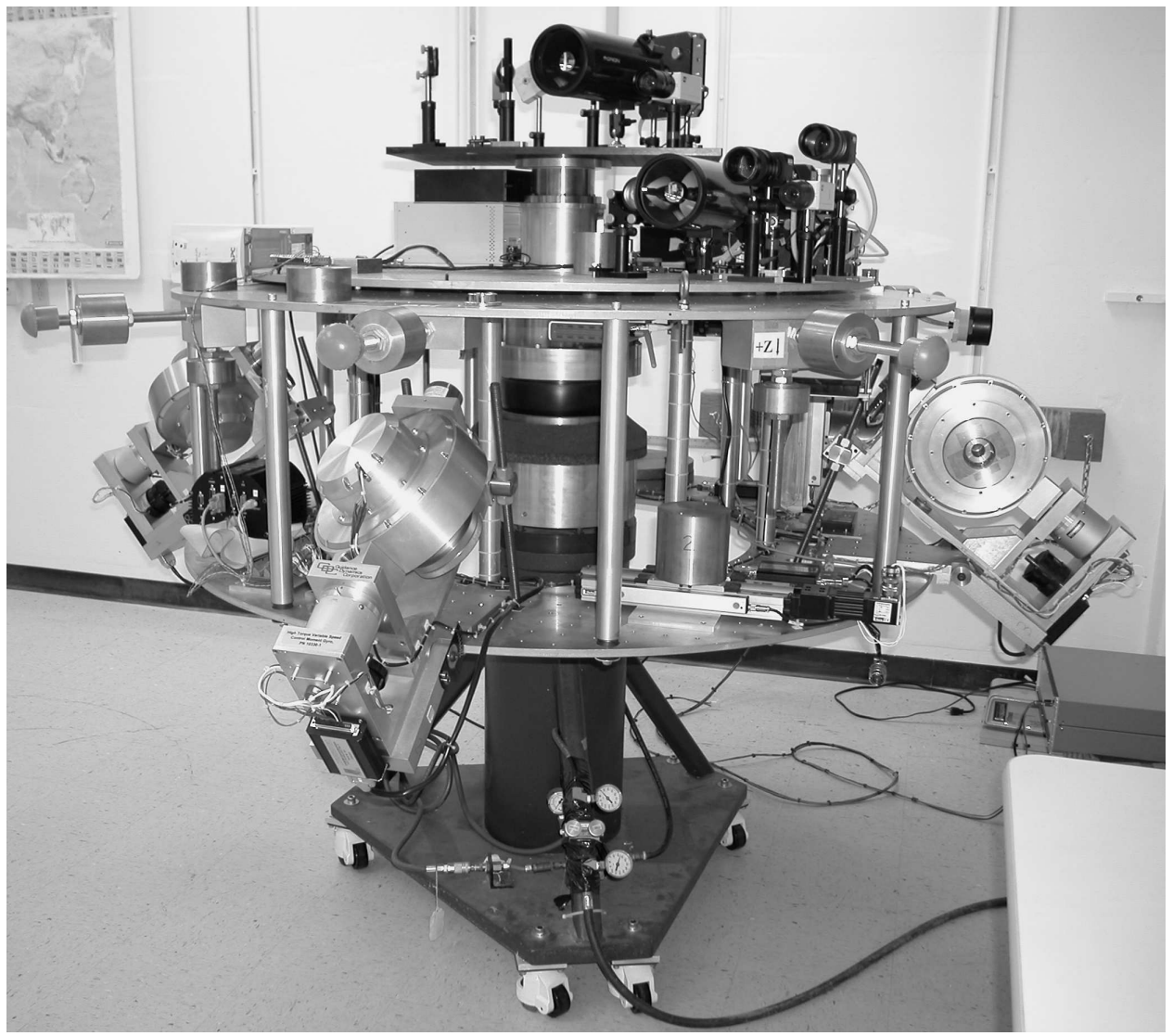

Figure 5. NPS Three-Axis Simulator 2 (TAS2)

be generated efficiently with the CMGs. For the BRMS, CMGs are preferable actuation devices due to the high speed slew capability requirement. The geometric configuration of the CMG array determines the characteristics of the momentum space for the spacecraft control. The CMG array has a inherent problem as CMG singularity. When the CMG gimbal angles are in the singularity state, the CMG array cannot produce a commanded torque. For the experimental verification of the automatic mass balancing methods, it is desirable to avid these singularity conditions. The CMG array configuration of TAS2 is shown in Figure 6. Considering the current configuration of TAS2 shown in Figure 6 with constant speed CMG operation (200

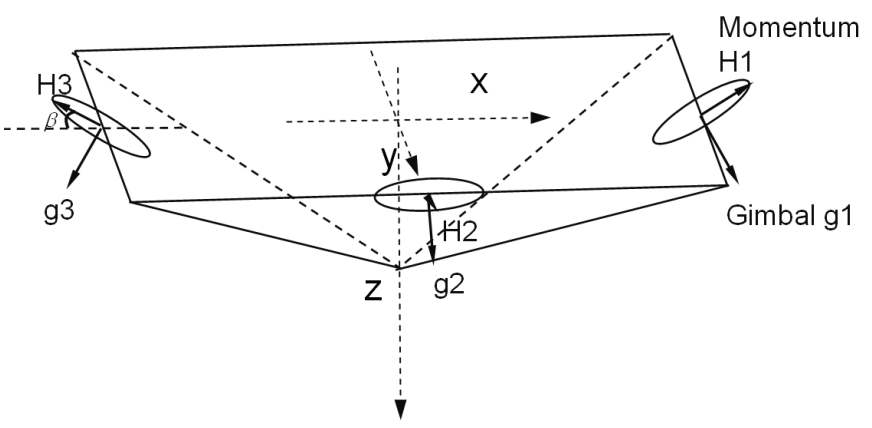

Figure 6. CMG Array Configuration of TAS2 
$\mathrm{rad} / \mathrm{sec}$ ), the total momentum from this CMG array geometry is written as

$$
\mathbf{h}=\left[\begin{array}{c}
-\cos \beta \sin \delta_{1} \\
\cos \delta_{1} \\
\sin \beta \sin \delta_{1}
\end{array}\right]+\left[\begin{array}{c}
-\cos \delta_{2} \\
\cos \beta \sin \delta_{2} \\
\sin \beta \sin \delta_{2}
\end{array}\right]+\left[\begin{array}{c}
\cos \beta \sin \delta_{3} \\
-\cos \delta_{3} \\
\sin \beta \sin \delta_{3}
\end{array}\right]
$$

where $\beta$ is the skew angle in Figure $6, \delta_{i}$ is the gimbal angle of the $i^{\text {th }}$ CMG $(i=1,2,3)$, and angular momentum from single CMG is normalized to $1 \mathrm{CMG}$ worth of momentum. Then the torque from the $\mathrm{CMG}$ array becomes the time derivative of the total CMG momentum, $\mathbf{h}$, which can be written as a matrix form such that

$$
\dot{\mathbf{h}}=A \dot{\delta}
$$

where,

$$
A=\left[\begin{array}{ccc}
-\cos \beta \cos \delta_{1} & \sin \delta_{2} & \cos \beta \cos \delta_{3} \\
-\sin \delta_{1} & -\cos \beta \cos \delta_{2} & \sin \delta_{3} \\
\sin \beta \cos \delta_{1} & \sin \beta \cos \delta_{2} & \sin \beta \cos \delta_{3}
\end{array}\right]
$$

The CMG steering law is a simple inversion of the matrix A such that

$$
\dot{\delta}=A^{-1} \dot{\mathbf{h}}
$$

When the matrix $A$ becomes singular, the steering law will not work. One way to deal with the CMG singularity without redundant CMGs is to use various singularity robust inverse methods. However, these techniques introduce small error in there commanded torque to move away from these singularity points. The introduction of this error may not degrade the performance for large slew angle maneuvers, where transition maneuver is of no concern. However, for automatic mass balancing system, it is more desirable to avoid these singularities without introducing error in the torque generated from the CMG array. Therefore, the CMG array should be configured to have the largest singularity free momentum envelop. Since the skew angle of each CMG can be changed with TAS2, various skew angles has been inspected. The optimal skew angle which provide maximum available singularity free momentum volume for CMG is found to be 90 degrees. Figure 7 shows the singularity surface of the $3 \mathrm{CMG}$ array with a 90 degree skew angle. When the magnitude of the momentum of the each $\mathrm{CMG}$ is $H_{C M G}$, this $\mathrm{CMG}$ array configuration provides singularity free momentum sphere with the radius of $H_{C M G}$. Attention is made such that the total momentum from the CMG array stays in this singularity free momentum sphere during the experiment.

TAS2 employs three linear stages with balancing masses on them. The three linear stages are aligned parallel to the three axis of the spacecraft body. Each linear stage has a maximum travel distance of $15 \mathrm{~cm}$ $( \pm 7.5 \mathrm{~cm})$ with an accuracy of $18 \mu \mathrm{m}$ and bidirectional repeatability of $\pm 5 \mu \mathrm{m}$. The maximum angular motion of the testbed with the spherical air-bearing in roll and pitch is 45 degrees. However, in order to protect from the instrumental and structural damages, a bumper with a structural damper is installed on the bottom of the spacecraft hub. Therefore, roll and pitch motion of the bus is limited to around 20 degrees. TAS2 is also equipped with Inertial Measurement Unit (IMU), two inclinometers (roll, pitch), on-board computer, IR sensor, and magnetometers for navigation. The IMU consists of 3 Fiber Optics Rate Gyroscopes with integrated 3 translational accelerometers.

The first experiment was carried out to determine the moment of inertia of the system and the center of the mass location. A sinusoidal reference trajectory has been generated and quaternion feedback control has been implemented for reference trajectory tracking. The testbed was reasonably well balanced manually to minimize momentum build-up and eventual saturation of the singularity free momentum space. With the recorded gimbal angles and spacecraft attitude data, the batch estimation of the system inertia matrix and center of mass vector is performed with the filtering method developed previously. The simple integration

filter $\left(\frac{1}{s}\right)$ is utilized for the linear least-square method. From the batch estimation, the resulting system inertia and center of mass vector become

$$
J=\left[\begin{array}{ccc}
61.7985 & -2.4520 & -15.2826 \\
-2.4520 & 56.2741 & -9.1634 \\
-15.2826 & -9.1634 & 215.1353
\end{array}\right], \quad m \mathbf{r}=\left[\begin{array}{c}
-0.0058 \\
0.0302 \\
0.4008
\end{array}\right]
$$




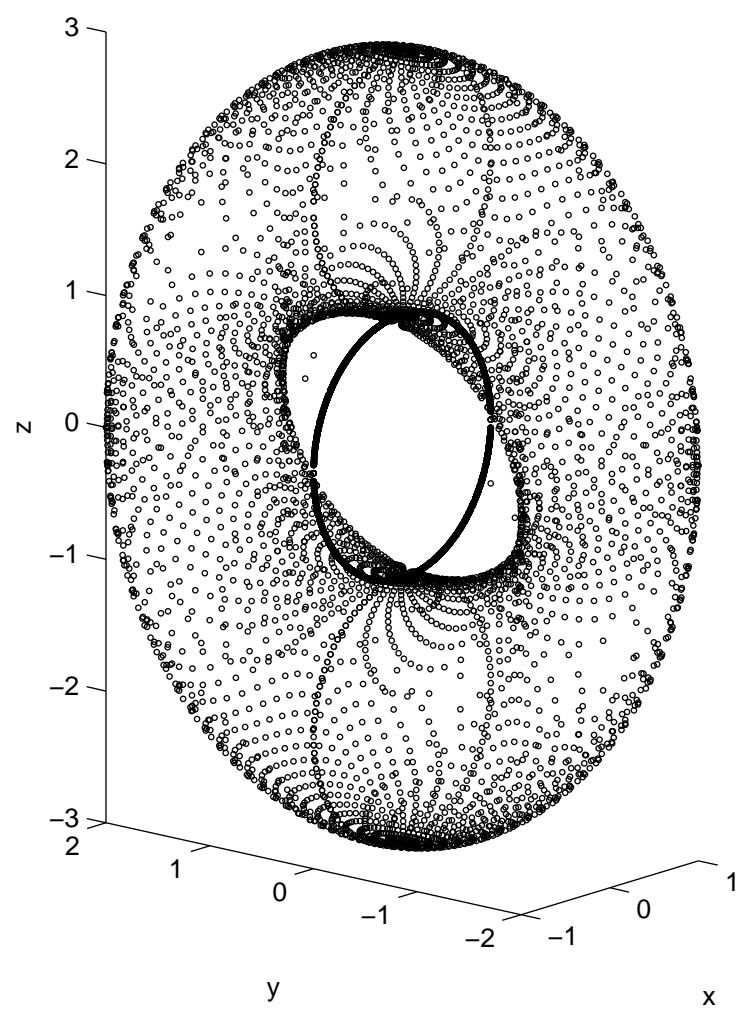

Figure 7. Singularity Surface of 90 degree skew with 3 CMG array

Although the direct compensation of the estimated center of mass showed improved balancing results, it still exhibited imbalance in some degree. As next step, the automatic mass balancing experiment with adaptive control method is carried out with the estimated inertia of the testbed. Figure 8 shows the actual attitude of the spacecraft during the adaptive mass balancing experiment. Sinusoidal attitude trajectory on roll and pitch of the spacecraft has been generated and the corresponding momentum trajectory has been used as a reference momentum trajectory of the adaptive mass balancing.

Figure 9 shows the experimental results showing the tracking error and balancing mass positions. Initially, the center of mass $z$-position was located lower than the center of rotation, while the $x-y$ position of the testbed was reasonably balanced manually. This is due to the fact that the $x-y$ direction of the center of mass offset can be easily checked by inspecting the roll and pitch angle at the equilibrium point. The left plot of Figure 9 shows that the tracking error is converging to zero. The actual positions of the balancing masses are also shown in the right plot. As anticipated, the balancing mass along the $\mathrm{z}$ direction has been lowered to correct the pendulum-like behavior, while the $x-y$ balancing masses has a smaller correction due to their better initial balancing. The testbed showed much improved performance compared to the direct compensation of the center of mass offset from batch estimation. The final balance was not perfect however, due to various reasons. First, the attitude measurements are not perfect. Friction and backlash in the CMG gimbal restrict the ability to command the desired net momentum state. Although the CMG flywheel assembly is reasonably balanced with respect to the gimbal axis, there still exists a center of mass offset for an individual CMG, which alters the center of mass of the whole testbed during the tracking control. However, the adaptive mass balancing control provides much improved balancing in reduced time compared to the manual adjustments.

\section{Conclusion and Future Research}

In this paper, a method of identifying the inertia property of a rigid body spacecraft as well as the center of mass location was investigated. The adaptive method for automatic mass balancing is also proposed for 

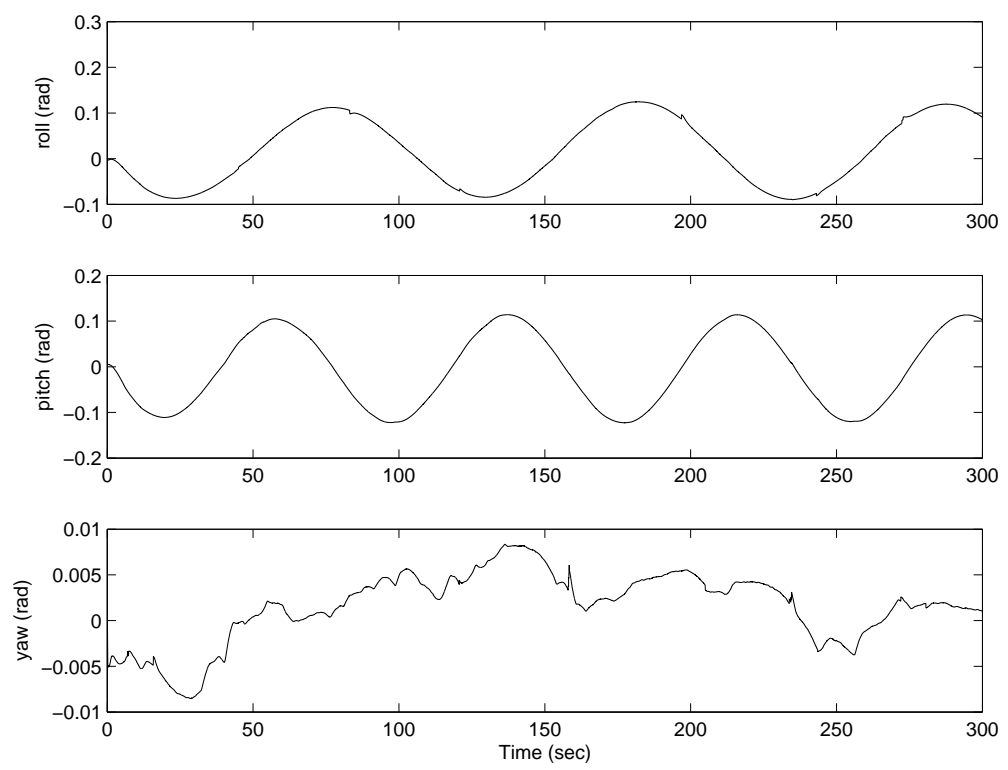

Figure 8. Attitude of the spacecraft during mass balancing
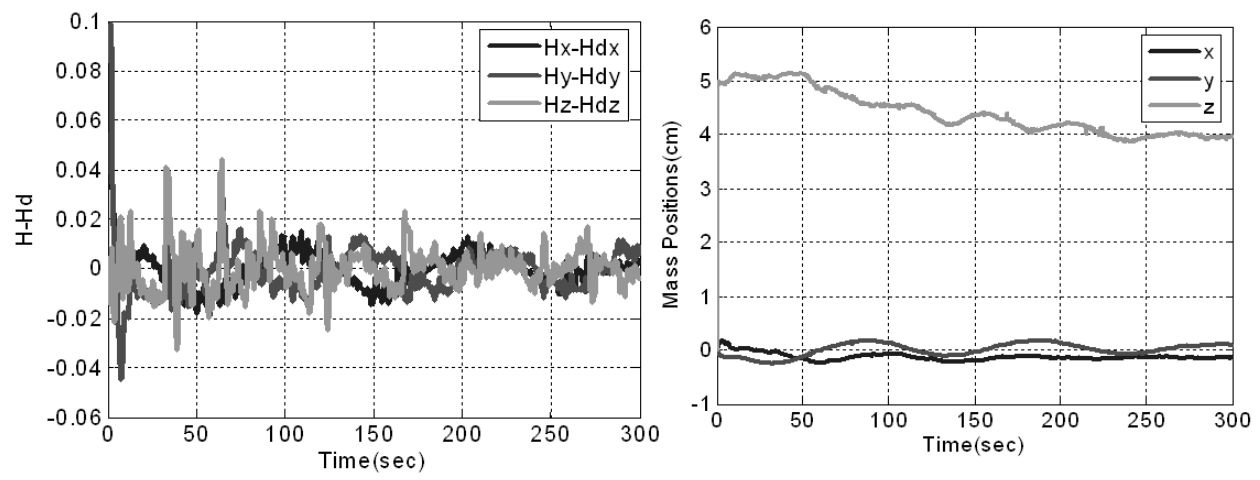

Figure 9. Automatic Mass Balancing Results from Experiments

online actuation of the balancing masses. For the verification of the proposed method, an experiment was also performed with the 3-axis rotational spacecraft testbed (TAS2). It is shown that the proposed adaptive actuation of the balancing masses can compensate the center of mass offset. The accuracy of the center of mass offset compensation can be improved with the proposed method with more accurate control of CMG gimbal and more accurate angular rate measurements.

\section{Acknowledgement}

Authors would like to thank Prof. Roberto Cristi of Naval Postgraduate School for helpful discussions.

\section{References}

${ }^{1}$ Schwartz, J. L., Peck, M. A., and Hall, C.D., "Historical Review of Spacecraft Simulators", Proceedings of the AAS/AIAA Spaceflight Mechanics Meeting, No. AAS 03-125, Ponce, Puerto Rico, Feburary 10-13, 2003.

${ }^{2}$ Chen, H., and Agrawal, B. "Methods of Slewing the Spacecraft to Minimize Slewing Time", AIAA Guidance, Navigation, and Control Conference and Exhibition, Monterey, California, August 5-8, 2002.

${ }^{3}$ Peck, M. A., and Cavender, A. R., "An Airbearing-Based Testbed for Momentum Control Systems and Spacecraft Line of Sight", 13th Annual AAS/AIAA Spaceflight Mechanics Meeting, San Antonio, Texas, AAS 03-127, February 2003.

11 of 12

American Institute of Aeronautics and Astronautics 
${ }^{4}$ Agrawal, B. N., Kim, J.-W., Romano, M., "Attitude Control and Determination of the NPS Bifocal Mirror Testbed," 55th International Astronautical Conference, Vancouver, Canada, 2004.

${ }^{5}$ Tanygin, S., and Williams, T., "Mass Property Estimation Using Coasting Maneuvers", Journal of Guidance, Control, and Dynamics, Vol. 20, No. 4, July-August 1997, pp. 625-632.

${ }^{6}$ Schwartz, J. L. and Hall, C. D., "System Identification of a Spherical Air-Bearing Spacecraft Simulator", proceedings of the 14th Annual AAS/AIAA Spaceflight Mechanics Meeting, February 8-12, 2004, AAS 04-122. 